\title{
ATRIBUTOS DEL PROBLEMA DINÁMICO DE CONTROL OPERACIONAL DE AGVs EN LA DISTRIBUCIÓN DE MATERIALES
}

\author{
Attributes of the dynamic problem of operational control \\ of agvs in the distribution of materials
}

EPISTEMUS

ISSN: 2007-8196 (electrónico)

ISSN: 2007-4530 (impresa)

\section{Isidro Ramos Torres 1}

Luis Felipe Romero Dessens 2

José Luis Martínez Flores 3

Elías Olivares Benítez 4

René Rogelio Durand Villalobos 5

Recibido: 21 de agosto de 2015

Aceptado: 22 de febrero de 2016

Autor de Correspondencia:

M. C. Isidro Ramos Torres

Correo: Isidro.Ramos@industrial.uson.mx

\section{Resumen}

En este trabajo se estudian los atributos del problema dinámico del control operacional de AGVs. Se discuten las implicaciones del uso de la información on-line como el principal elemento del carácter dinámico de los problemas. Seutiliza la taxonomía de Psaraftis para identificar los atributos (evolución, calidad, disponibiliad, y procesamiento) de la información utilizada. Se obtuvieron resultados parciales sobre las tendencias de los estudios dinámicos y sus características.

Palabras clave: Distribución de materiales, control operacional, AGV, información tiempo-real.

\section{Abstract}

This work explores the attributes of the dynamic problem of operational control of AGVs. Implications of use of the information on-line as the main element of the dynamic nature of the problems are discussed. The Psaraftis taxonomy is used to identify the attributes (evolution, quality, availability, and processing of information) in the solution on problems of operational control of AGV. Partial results on trends in dynamic studies and their characteristics were obtained.

Key words: Material distribution, operational control, AGV, on-line Information.

Depto. de Ingeniería Industrial, Universidad de Sonora / Correo: isidro.ramos@industrial.uson.mx 1 Depto. de Ingeniería Industrial, Universidad de Sonora / Correo: Iromero@industrial.uson.mx 2 Universidad Popular Autónoma del Estado de Puebla / Correo: joseluis.martinez01@upaep.mx 3 Universidad Popular Autónoma del Estado de Puebla / Correo: elias.olivares@upaep.mx 4 Depto. de Ingeniería Industrial, Universidad de Sonora / Correo: rdurand@industrial.uson.mx 5 


\section{INTRODUCCIÓN}

La distribución de materiales (MD, por sus siglas en inglés) es una de la actividades del manejo de materiales que implica mover el material correcto al lugar correcto, en la cantidad necesaria, en secuencia y correcta posición o condición para minimizar los costos de producción [1] [2]. La satisfacción de las funciones de la MD se logra resolviendo los problemas relacionados al Diseño y Control de Sistemas de AGVs [3].

En ambientes de manufactura, el problema de la MD plantea la necesidad de involucrar múltiples vehículos, en este caso Vehículos Automáticos Guiados (AGVs), determinar y administrar rutas, resolver problemas de conflictos de tráfico, determinar la magnitud de la carga y el número de viajes de los AGVs, ubicar puntos de carga y descarga ( $P / D$, por sus siglas en inglés), puntos reposo de AGVs, diseño de rutas mediante particiones (tándem o zonas), y diseño de topologías (circuitos o mallas) unidireccionales o bidireccionales, entre otros [3].

Se han desarrollado una gran cantidad de planteamientos y propuestas metodológicas de solución para los diferentes problemas desde los años 1950's desde una perspectiva estática y posteriormente a través de una perspectiva dinámica con la colaboración de las tecnologías de información desarrolladas y difundidas en los ámbitos industriales. Esta posibilidad cada vez más pronta y eficiente de obtener y procesar la información (online) propicia que dicha información pueda ser utilizada con inmediatez en la toma de decisiones.

El presente trabajo tiene como objetivo identificar los aspectos dinámicos/estáticos en los problemas de control (asignación, programación y ruteo) de AGVs en MD, abordados en la literatura en ambientes de manufactura.

La taxonomía de Psaraftis [4] es utilizada para caracterizar la información como fuente principal de la dinámica en el enfoque del planteamiento. Se eligieron 10 artículos publicados entre el 2012 al 2014, de los cuales se extrajeron los atributos de la información, el enfoque y la metodología de solución al problema planteado.

\section{ANTECEDENTES}

\section{La información: fuente de la dinámica}

La dinámica en los problemas de programación y ruteo de vehículos de transporte ha sido caracterizada por Psaraftis, como un escenario favorecido por los adelantos tecnológicos en comunicación y procesamiento de datos, aunado a las tendencias de eficiencia en la logística de distribucion de productos y materias primas en la creciente apertura de los mercados. Las tecnologías implicadas en esta evolucion de la logística de distribucion, se atribuyen inicialmente al Intercambio Electrónico de Datos, Sistema de Posicionamiento Global, Sistema de Información Geográfica, y Vehículos Inteligentes (AGV). Estas tecnologías permiten la operación y control en tiempo real (on-line) del sistema, la reducción de costos, la integración de componentes tecnológios y rapidez en el procesamiento de la información [4]. Psaraftis establece que un problema es denominado dinámico, "si la información para la determinación de una ruta requiere actualización a través del tiempo" [4].

\section{Qué son los AGVs}

Los vehículos automáticos guiados (AGV, pos sus siglas en ingles), son vehículos controlados por computadora con capacidad de carga. Estos vehículos son energizados por baterías y transportan material sin necesidad de un operador. Se tienen definidos caminos (rutas-guía) o áreas dentro de o sobre los cuales pueden transitar. Este movimiento dirigido se logra por uno o varios medios, siguiendo una trayectoria definida, guiado por cables enterrados inductivos, bandas magnéticas u ópticas, o alternativamente a modo de guía inercial o láser.

Los AGVs pueden tener diferentes denominaciones como: Automatic Guided Carts (AGCs), Robot móviles o Lift trucks híbridos [5].

\section{Problemas del control opeacional de AGVs}

El control operacional mantiene como principal objetivo satisfacer la demanda de materiales tan rápido como sea posible sin la ocurrencia de conflictos entre AGVs. Las actividades básicas para la operación y control del sistema de distribución son: a) Asignación de tareas de transporte (dispatching), b) Selección de ruta (routing), y c) Programación y secuenciación de AGVs (schedulling).

El problema de control operacional en sistemas de distribución con AGVs afronta el mismo escenario que el problema del agente viajero múltiple (mTSP) ó problema de ruteo de vehículos (VRP); sin embargo, la operación de los vehículos en espacios pequeños (comparado al problema mTSP ó VRP) como centros de trabajos (floor shop), bodegas de almacenamiento o terminales de contenedores, presenta otras situaciones que tienen impacto en el desempeño del sistema de distribución y deben ser consideradas, tales problemas son: a) El espacio dispuesto para el tránsito de los AGVs (maniobra carga y descarga), b) La posibilidad de congestionamiento de rutas, colisiones, c) La distancia y tiempo pueden no tener correspondencia y se requiere el trazado de rutas [6], y d) Aspectos de funcionamiento y control (programacion) de las unidades de AGV, entre otras. Los problemas a resolver derivados de las consideraciones anteriores, son múltiples, podemos mencionar algunos problemas integrados a los problemas básicos de asignación, programación y ruteo de AGVs; estos enfoques pueden dirigirse a:

* Establecer mecanismos para evitar o reducir las sobrecargas de rutas, bloqueos y/o colisiones con otros AGVs.

* Administrar la distribución de planta, estableciendo de manera fija o dinámica localidades como: rutas-guía mediante formación de tándem, zonas independientes, circuitos, y mallas, entre otras topologías. 
* Ubicación de lugares de espera de AGVs (I/D, por sus siglas en inglés), ubicación de puntos de carga y/o descarga (P/D), así como ubicación de lugares de almacenamiento de materiales (WIP) y sus capacidades.

* Identificar métricas apropiadas que representen eficazmente el desempeño de los sistemas.

\section{EL EFECTO DE LA INFORMACIÓN}

\section{SOBRE EL CONTROL OPERACIONAL}

La disposición de la información para tomar decisiones en la operación de AGVs, es determinante en la naturaleza del control ejercido sobre dichas actividades. Cuando se conocen previamente el origen, destino, tiempo de ejecución y tiempo de transporte de una tarea de distribución, es posible establecer control estático (offline). En otro caso, debido a la naturaleza estocástica de los procesos de transporte es necesario establecer sistemas de control capaces de facilitar decisiones en tiempo real (online). El control on-line puede ser ejercido desde un tablero de control de AGVs único (control centralizado) y mediante el control descentralizado, el cual ubica varios puntos de control en el sistema. En la situación de control off-line, los vehículos pueden ser asignados a las cargas de una manera óptima mediante un planteamiento de problema de asignación [3].

\section{Asignación de AGVs}

La asignación (dispatching) es la regla utilizada para seleccionar un vehículo de transporte de materiales. Esta actividad puede ser realizada desde dos puntos de vista: asignar un carga a un vehículo disponible para su transporte $(\mathrm{W}-\mathrm{I}$, por sus siglas en inglés) ó asignar una carga a un vehículo no disponible (V-I, por sus siglas en inglés) [3]. En Egbelu y Tanchoco [7] se presenta una caracterización de las reglas heurísticas de asignacion para W-I. Sin embargo, gran parte de las reglas referidas, tanto en el criterio W-I como V-I, dependen de la información disponible y su evolución (on-line/off-line).

\section{Ruteo de AGVs}

El ruteo es la elección de la ruta o segmento de ruta por la cual se transportará una carga desde el punto de abastecimiento hasta su destino. Los enfoques para resolver el problema de ruteo son: ruteo estático y ruteo dinámico. El modelo estático, considera la ruta desde un origen a un destino anticipadamente tomando la ruta más corta. En el ruteo dinámico la decisión de rutas se basa en la información en tiempo real sobre el tráfico de vehículos en la red de distribución, por lo tanto existe la posibilidad de elegir segmentos de ruta más apropiados en ese momento [3].

\section{Programación y secuenciación de AGVs}

La programación y secuenciación de los AGVs son actividades que también dependen de la información de los estados del sistema, principalmente aspectos de tráfico, disponibilidad de vehículos y requerimientos de demanda. La programación determina el tiempo de salida o llegada de un AGV, de cualquier nodo de segmento de una ruta, nodo de intersección o punto de carga o descarga (P/D) [3].

\section{TAXONOMÍA DE PSARAFTIS}

El modelo presentado por Psaraftis [4] es una taxonomía de 5 elementos y fue utilizada para caracterizar la información de entrada en la solucion de problemas de Ruteo de vehículos. Los atributos de la taxonomía se despliegan en la figura 1.

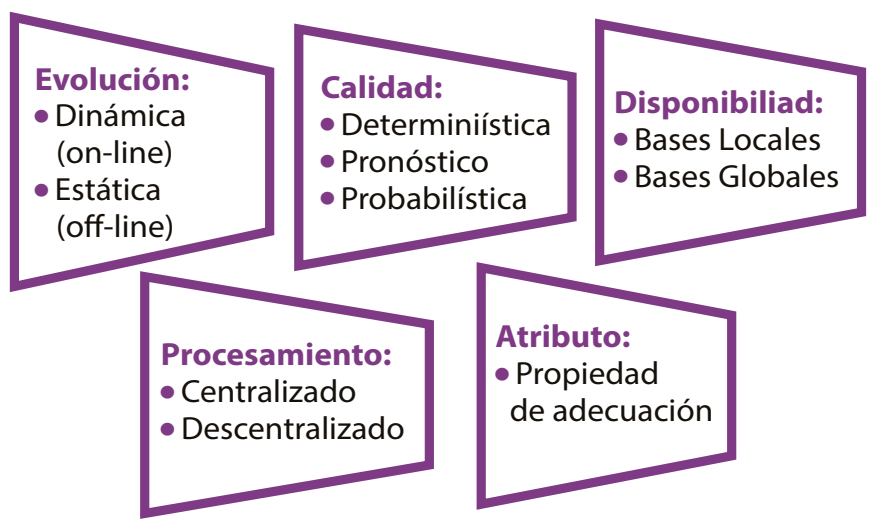

Figura 1. Elementos de la taxonomía de Psaraftis.

Evolución de la información. Tiene la función de determinar si la información de entrada es estática o dinámica. Se considera información dinámica si es actualizada constantemente y no tiene cambios en el tiempo durante el proceso de decisión. El caso estático implica el previo conocimiento de la información y su permanencia sin cambio en el tiempo. Cuando la información tiene un comportamiento probabilístico pero conocido (que no requiere actualizacion durante el tiempo de ruteo), podemos decir que es un caso estocásticoestático [4].

\section{Calidad de la información}

Parte o toda la información puede ser considerada como:

* Determinística. Si no tiene cambio durante el proceso de decisión.

* Pronóstico. Alguna información no se conoce con certeza, sólo como un pronóstico.

* Probabilística. La información sigue alguna distribución de probabilidad o se involucra en algún proceso estocástico. La calidad de la información es definida para un punto específico en el tiempo en el que se toma la decisión.

\section{Disponibilidad de la información.}

La información puede provenir de:

* Bases locales. La fuente de la información se encuentra en localidades únicas. 
* Bases globales. La información puede estar disponible de uno más elementos de una red (EDI).

\section{Procesamiento de la información}

El procesamiento puede ser posible mediante dos esquemas en cuanto a cómo se procesa la información disponible. Los esquemas son:

* Centralizado. La información es colectada y procesada en una unidad (programador humano, hombremáquina asistido por computador, o un sistema totalmente automatizado).

- Descentralizado o parcialmente centralizado. En este esquema la información se distribuye a distintos elementos del sistema para ser procesada localmente.

* Atributo. El atributo es una propiedad de adecuación. Algunas metodologías de solución son más apropiadas para esquemas de procesamiento descentralizado de solución tales como esquemas de partición o de descomposición.

\section{RESULTADOS DE REVISIÓN DE LITERATURA}

\section{Problemas dinámicos encontrados}

En Conffesore et al. [8] se plantea el problema de asignación de AGVs (vehículo-iniciado), mediante un modelo de redes de flujo utiliza un sistema de decisión que actualiza en el tiempo los trabajos futuros de transporte y el estatus de posiciones de AGVs. En Chiew y Kin [9] se actualizan las predicciones de la posición de los vehículos para reducir conflictos de tráfico. En tiempo real y mediante un algoritmo de programación AGVs en Erol et al. [10] se presenta un enfoque multi-agente para programar máquinas y AGVs simultáneamente. En Udhayakumar y Kumanan [11] se plantean el problema de la programación de la producción, programación y ruteo dinámico de AGVs, para lo cual se utiliza un algoritmo de optimización ACO y PSO. De manera similar en Tuma et al. [12] se integra la programación de producción y AGVs, mediante la hibridación de GA+TS soportado en un sistema de banderas.

\section{Problemas estáticos encontrados}

En el problema de programación de máquinas AGVs, Lacomme et al. [13] se proponen un modelo redes, así mismo en Zeng et al. [14] se plantea un algoritmo en dos fases: heurístico y búsqueda local (tabú), con el objetivo de minimizar los bloqueos de máquinas. También en Badakhshian et al. [15] se utiliza un algoritmo genético (GA, por sus siglas en inglés) controlado por lógica difusa. Como una estrategia de evasión de conflictos de tráfico y para resolver el problema de asignación, en Zheng et al. [16] se propone un modelo de rutas por zonas independientes. También Nishi y Tanaka [17] resuelven el problema de asignación y ruteo con un modelo que utiliza redes de Petri de solución óptima.

Tabla 1. Identificación de los atributos de la información en la literatura revisada.

\begin{tabular}{|c|c|c|c|c|c|c|c|c|c|c|c|c|c|c|c|c|c|c|c|c|c|c|c|c|c|c|}
\hline \multirow[b]{2}{*}{ 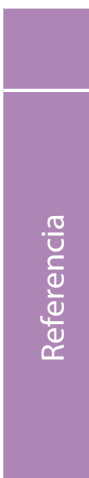 } & \multicolumn{5}{|c|}{$\begin{array}{c}\text { Problemas } \\
\text { tratados }\end{array}$} & \multicolumn{11}{|c|}{ Atributo: Método de Solución } & \multicolumn{2}{|c|}{ Evolución } & \multicolumn{4}{|c|}{ Calidad } & \multicolumn{2}{|c|}{$\begin{array}{l}\text { Disponi- } \\
\text { bilidad }\end{array}$} & \multicolumn{2}{|c|}{$\begin{array}{c}\text { Procesa- } \\
\text { miento }\end{array}$} \\
\hline & 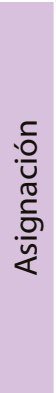 & 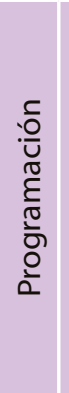 & 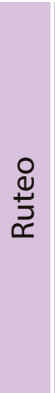 & 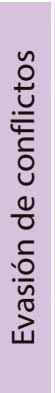 & 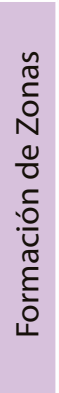 & 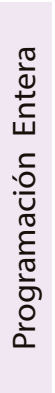 & $\begin{array}{l}\frac{0}{3} \\
\frac{\pi}{4} \\
\frac{d}{0} \\
\tilde{y} \\
\frac{\tilde{U}}{\tilde{U}} \\
\simeq\end{array}$ & 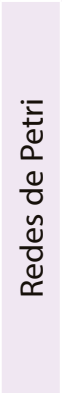 & 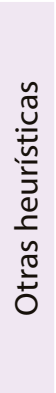 & 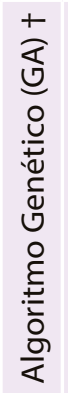 & 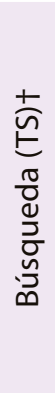 & 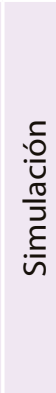 & 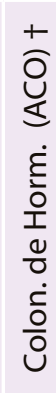 & 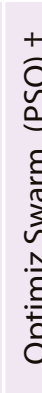 & 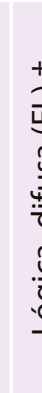 & 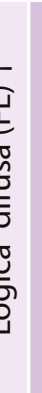 & 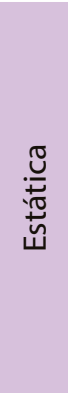 & 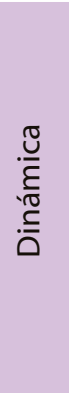 & 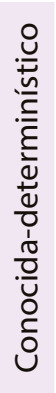 & 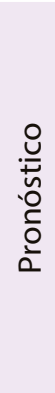 & 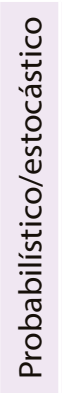 & 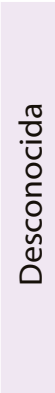 & 厄ั & $\begin{array}{l}\bar{\pi} \\
\stackrel{0}{0} \\
\text { 은 }\end{array}$ & 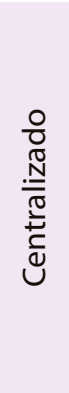 & 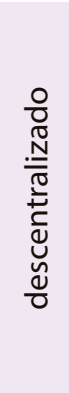 \\
\hline [9] & & * & * & * & & & & & * & & & * & & & & & & * & & & * & & * & & * & \\
\hline [17] & $*$ & * & * & * & & & & * & * & & & & & & & & * & & $*$ & & & & * & & * & \\
\hline [8] & * & * & & * & & * & * & & * & & & & & & & & & * & & * & & & * & & * & \\
\hline [16] & * & * & * & * & * & & & & & & & $*$ & & & & & * & & * & & & & * & & * & \\
\hline [11] & & * & & & & & & & & & & & * & * & & & & $*$ & & & $*$ & & * & & * & \\
\hline [12] & & * & & & & & & * & & * & $*$ & & & & & & & * & * & & & & - & - & $*$ & \\
\hline [15] & & * & & & & & & & & * & & & & & * & & * & & $*$ & & & & & * & $*$ & \\
\hline [10] & & $*$ & & & & & & & & * & & & & & $*$ & & & $*$ & * & & & & & * & $*$ & \\
\hline [14] & & * & * & & & * & & & * & & * & & & & & & * & & * & & & & * & & * & \\
\hline [13] & & $*$ & & & & & $*$ & & & * & $*$ & & & & & & * & & & * & & & * & & $*$ & \\
\hline
\end{tabular}




\section{Resumen características de la información}

* Los atributos de la información y el problema abordado en la literatura se colectaron en la tabla 1. En ésta se observa que:

* Existen algunos enfoques integrales en las investigaciones, es decir, se abordan varios problemas del control operacional y de otra índole.

* Se encontraron casos de evolución dinámica y estática en proporciones semejantes.

* En la mayoría de los casos se utiliza información determinística conocida, en menor proporción información pronosticada o probabilística.

* La disponibilidad de la información en mayor proporción es de tipo local y bajo un control centralizado.

\section{CONCLUSIONES}

La información en tiempo-real es el elemento principal que posibilita a los investigadores modelar escenarios más adaptados a las necesidades dinámicas. La disposición, la rapidez de procesamiento, el intercambio de datos, el grado de integración de las tecnologías de información (hardware y software), el uso de tecnologías de posicionamiento e identificación, y la implementación de vehículos automáticos guiados (AGVs), son los elementos que favorecen la dinámica en los planteamientos de problemas.

El concepto de problema dinámico de Psaraftis, es difícil de lograr en la práctica, por ello algunos investigadores definen su planteamiento de problema como dinámico a pesar de que en sentido estricto se soluciona un problema estatico cuya actualización se realiza cada pequeño periodo de tiempo, de tamaño insignificantes en relación al tiempo requerido para percibir un posible cambio de estado del sistema.

En el ejercicio de caracterización de los atributos de la literatura, se observó que (sin ser una muestra representativa) los artículos tratan problemas dinámicos y estáticos en proporciones semejantes. Mayormente, los problemas utilizan información de fuentes locales y determinísticas.

Los problemas de asignación, secuenciación y programación de AGVs, son susceptibles a las características de la información, así como al problema de ruteo; sin embargo, el ruteo mantiene su dinamismo desde su planeación y durante la ejecución, es decir que existe la posibilidad de re-ruteo soportado por la información en tiempo-real.

En futuras investigaciones, se podrían establecer hipótesis relativas a correspondencia de los atributos: evolución, calidad, disponibilidad, procesamiento y metodología, entre sí, o con atributos de otra índole, con el objetivo de identificar tendencias o patrones de orientación hacia donde se dirigen las investigaciones.

\section{BIBLIOGRAFÍA}

[1] M.P. Stephens y F. E. Meyers," Manufacturing Facilities Design \& Material Handling", 4 ed., Perason Prentice Hall,, 2010.

[2] ASME, (American Society of Mechanical Engineers); IMMS, (International Material Management Society);, "Materials Handling Handbook", 2da ed., R. A. Kulwiec, Ed., Wiley, 1985.

[3] I. F. A. Vis, «Survey of research in the design and control of automated guided vehicle systems,» European Journal of Operational Research, n 170, pp. 677-709, 2006.

[4] H. N. Psaraftis, «Dynamic vehicle routing: Status and Prospects,» Annals of Operations Research, n 61, pp. 143164, 1995.

[5] B. Trebilcock, «What is an AGV?,» Modern Material Handling, pp. 22-28, Junio 2011.

[6] L. Qiu, W.-J. Hsu, S.-Y. Huang y H. Wang, «Scheduling and routing algorithms for AGVs: a survey,» International Journal Production Research, vol. 40, no 3, pp. 745-760, 2002.

[7] P. J. Egbelu y J. M. A. Tanchoco, «Characterization of automatic guided disptaching rules,» International Journal of Production research, vol. 22, n 3, pp. 359-374, 1984.

[8] G. Confessore, M. Fabiano y G. Liota, «A network flow based heuristic approach for optimizing AGV movements,» Journal Intelligent Manufacturing, n²4, pp. 405-419, 2013.

[9] K. Chiew y S. Qin, "Shedulling and Routing of AMOs in an Intelligent Transport System,» IEEE Transactions on Intelligent Transportation Systems, vol. 10, n³, pp. 547-552, 2012.

[10] R. Erol, C. Sahim, A. Baykasoglu y V. Kaplanoglu, «A multi-agent based approach to dynamic scheduling of machines and automated guided in manufacturing systems,» Applioed Soft Computing, n 12, pp. 1720-1732, 2012.

[11] P. Udhayakumar y S. Kumanan, «Integrated scheduling of flexible manufacturing system using avolutiionary algorithms,» International journal on manufacturing Technology, n 61, pp. 621-635, 2012.

[12] C. C. Tuma, O. J. Morandin y V. F. Caridá, «Minimizing the makespan for the problem of reactive production scheduling in a FMS with AGVs using a new structure of chromosome in a hybrid GA with TS,» de 18 th Conference on Emerging Technologies \& Factory Automation (ETFA), Cagliari, 2013.

[13] P. Lacomme, M. Larabi y N. Tchernev, «Job-Shop based framework for simultaneous shceduling of machines and automated guided vehícles,» International Journal Economics, no 143, pp. 24-34, 2013.

[14] C. Zeng, J. Tang y C. Yan, «Sceduling of no buffer job shop with blocking constrains and automated guided vehicles,» Applied Soft Computing, n² 24, pp. 1033-1046, 2014.

[15] M. Badakhshian, S. B. Sulaiman y M. K. A. Ariffin, «Performanceoptimization of simultaneous machine and automated guided vehicle sheduling using fuzzy logic controller based genetic algorithm,» International Journal of Physical Sciences, vol. 7, no 9, pp. 1461-1471, 2012.

[16] H. Zheng, D. Tang, W. Gu y M. Dai, «Distributed control of multi-AGV system based on regional control model,» Production Engineering Research and Development, $n^{\circ} 7$, pp. 433-441, 2013.

[17] T. Nishi y Y.Tanaka, «Petri Net Decomposition Approach for Dispatching and Conflict-Free routing of Bidirectional Automated Guided Vehicle Systems,》 IEEE Transactions on Systems, Man and Cybernetics- Part A: Systems and Humans, vol. 42, n 5, pp. 1230-1243, 2012. 